\title{
Satellite Space Information Network: Application and Prospect
}

\author{
Weigui Zhou, a, Hong Ao ${ }^{1, b}$, Quan Zhou ${ }^{1, c}$, Yuan Gao ${ }^{1,3, d}$ and $\mathrm{Yi} \mathrm{Li}^{2,}$ e $^{*}$ \\ ${ }^{1}$ Xichang Satellite Launch Center, Sichuan, China \\ ${ }^{2}$ The High School Affiliated to Renmin University of China, Beijing, China \\ ${ }^{3}$ China Defense Science and Technology Information Center, Beijing, China \\ awgzhou@nudt.edu.cn, baohong76@aliyun.com, czhouquanxslc@foxmail.com \\ dyuangao08@gmail.com, ${ }^{\mathrm{e}}$ liyi@rdfz.cn \\ * The corresponding author
}

Keywords: Satellite; Space information network; Structure; Analyze

\begin{abstract}
Spatial Information Network is a platform for the carrier space, real-time acquisition, transmission and processing of spatial information network system, which includes a space platform geosynchronous satellites in low orbit satellite, stratospheric balloon, manned or unmanned aerial vehicles. Different types of satellite orbit performance can be formed to cover the global space-based network, stratospheric balloon, manned or unmanned aircraft, airships, etc. can be formed to meet specific uses space-based network. With the terrestrial Internet (e.g. Foundation Network) continues to develop, the space-based, airborne, ground and other basic platforms constitute the integrated network seamlessly connected to each other, to achieve any user anywhere in the world over the Internet, it has become the next generation Internet an important part of the study, but also key technologies for military battlefield communications.
\end{abstract}

\section{Introduction}

Spatial Information Network is a space motion platform (including satellites, airships and aircraft) as the carrier, real-time access, network transmission and processing spatial information, can support up deep space exploration [1], down to support applications such as earth observation, which will human sciences, culture and production activities to expand the space, far from the sea, and even deep space, is a global hotspot [2].

Backbone Communications Networks uses a high-capacity links to connect the main nodes information networks bear voice, video, Internet and other data gathering and diversion, plays an important role in the communication system. You can use a relatively low rate between major nodes and users, but must have the ability to multi-user access [3]. Fig. 1 is a schematic overview of an empty earth integrated network backbone transmission network. Space-based network between space-based and ground network plays a connecting role, is empty earth integrated network essential part of the future, and the high-speed, flexible, all-weather mobile space-based backbone [4] transmission network is an important part of this realization infrastructure. In conventional terrestrial telecommunications infrastructure, the backbone transmission network mainly depends on single-mode fiber as its primary data link. However, the empty earth integrated network cannot rely solely on fixed infrastructure, but also a need for a capacity equivalent to the optical fiber, and may play a role in the mobile backbone transmission network at any place and in any weather conditions. Early airspace link uses radio frequency, which is not affected by weather conditions, but the limited transmission capacity.

Spatial Information Network in comparison with the ground network has the following outstanding features [5]:

(1) Covering a wider range of terrestrial networks currently only cover land, sea or less than $20 \%$, and space information network global coverage and even outer space. Spatial information network and 
therefore need to adapt to complex spatial and geographical environment, the traditional network of information technology cannot meet the above requirements.

(2) The application of space applications cover a wider field of space observation information networks, information transmission, processing and application of human entry, understanding, use of space means of support, but also gave birth to major scientific discoveries, technological inventions, as well as strategic emerging industries carrier, has become the information sciences, earth sciences and mathematical sciences interdisciplinary development of an important frontier direction.

(3) Due to technical difficulties greater span of time and space, high dimensional information, spatial information transmission network communications, real-time information processing capacity limit of a challenge. Meanwhile, the extremely limited time and space constraints and space resources, capacity to become prominent spatial information network bottlenecks, and optimize the use of their planning often cannot use the traditional network theory and techniques. Furthermore, the special requirements of high reliability, space facilities, the traditional Internet, telecommunication network technology should not even be directly applied to spatial information network.

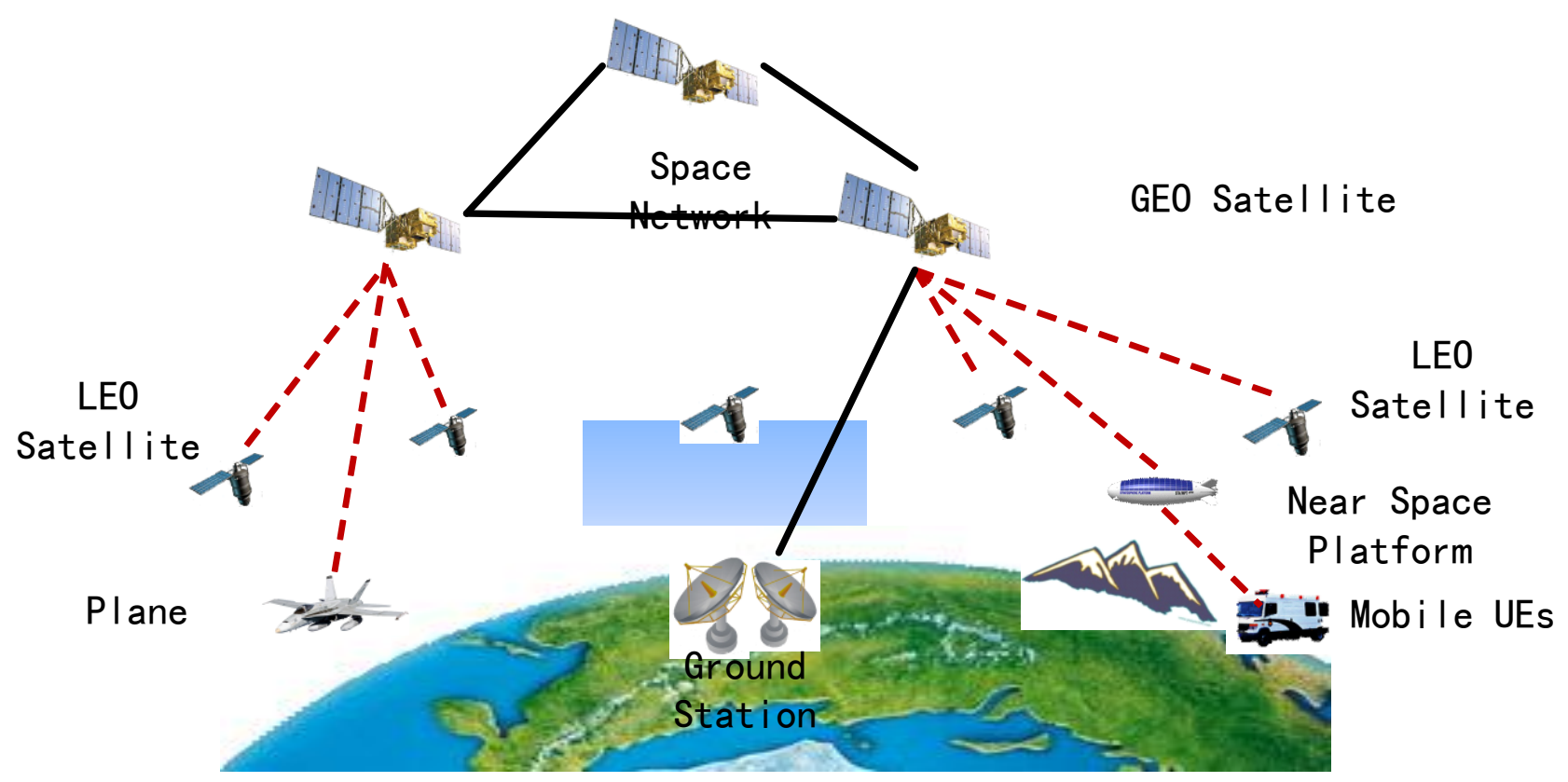

Figure 1. Finite element calculation model of arch dam and bedrock

\section{Development of the USA}

The most famous space-based transmission network Common Data Link (CDL), dominated by the US Department of Defense developed in the early 1990s, the Ku-band (12 18 GHz), the highest rate of up to $274 \mathrm{Mb} / \mathrm{s}$, communication distance of up to hundreds of kilometers [6], to provide full-duplex, point to point communication link between an aircraft and ships as well as aircraft and ground between bases, airspace control platform. Common Data Link improved (Advanced Common Data Link, ACDL) using Ka-band (26 $40 \mathrm{GHz}$ ), the transmission rate up to $3 \mathrm{~GB} / \mathrm{s}$.

In order to further improve the transmission rate, the US Defense Advanced Research Projects Agency (DARPA) has implemented a "free-space optical test network pilot project" (FOENEX) in 2010 and 2012. The program makes the rate of the mobile communication network backbone to reach $10 \mathrm{~GB} / \mathrm{s}$, using WDM (Wavelength Division Multiplex), the rate of up to $100 \mathrm{~GB} / \mathrm{s}$. For space-based and space-based network, free-space optical communication (Free Space Optical Communication, FSO) is the first choice of the composition of the mobile backbone transmission network, not only having a communication capacity is equivalent to the optical fiber, and long-distance work. It is foreseeable that free-space optical communications will play a major role in the space-based network. 
However, it cannot be transmitted through the clouds, which means it has about 40 percent of the time not well functioning, thus leading to network availability low.

In order to achieve all-weather high-speed mobile backbone transmission network, the US Defense Advanced Research Projects Agency in early 2013 to develop a "100G radio backbone transmission network plan" [7]. The goal of the program is to design and build a communication link and having airborne optical communications equivalent capacity and same distance from the action. The link can transmit through the clouds and high availability, system capacity of $100 \mathrm{~Gb} / \mathrm{s}$. Air-link communication distance of at least $200 \mathrm{~km}$, to-Earth link (height of 60,000 feet) communication distance of at least $100 \mathrm{~km}$. The link is in keeping tactics related throughput and link distance must be available around the clock (including clouds, rain, fog and other weather conditions). Its system size, weight and power consumption depending on the placement platform (mainly long-life high-altitude airspace platform). US Defense Advanced Research Projects Agency that the use of millimeter wave communication system is to achieve "100G radio backbone transmission network plan," [8] the best choice. There are more than $10 \mathrm{GHz}$ millimeter-wave frequencies of the spectrum, the system can meet the spectrum requirements. Transmit power can be increased by combining high power amplifier array antenna. Cumulus atmosphere and the total loss is only about 10dB. Multi-channel transmission can also use multiple input multiple output technology line of sight (LOS MIMO) to achieve.

Horizon MIMO technology uses multiple transmit and receive antennas to achieve space division multiplexing the head. When the transmission distance is less than a distance equal to the Rayleigh (Rayleigh Range), the received signal by appropriate signal processing to decode multiple independent information. Antenna spacing of 10 meters, the Rayleigh distance [9] of up to 100 kilometers. "100G radio backbone transmission network plan" recommends using a combination of various techniques to achieve performance targets. First, to ensure that overcoming the permissible atmospheric fading to achieve under the premise of long distance using millimeter wave spectrum to provide sufficient bandwidth. Secondly, the use of higher order modulation spectrum efficiency. Third, the use of multi-polarization and space division multiplexing multi-channel.

\section{Status of China}

According to China's "national civil space infrastructure and long-term development plan" in the next ten to fifteen years, China will follow the integration of the empty world of ideas, development of space infrastructure [10], the development of world air-speed Internet system. On the basis of the national civil space infrastructure, based on the follow the international development trend of empty earth integrated information network deep integration of building networking, global coverage, broadband services and mobile satellite communication system security, and in particular the fifth terrestrial broadband network generation mobile communication systems, Internet integration, the formation of independent air earth integrated information network.

Since 2008, Shanghai Institute of Microsystem millimeter wave communications technology research carried out. In 2012, using a large off-axis parabolic mirror built $2.4 \mathrm{~m}$ communication link. Experimental results show that the maximum transmission rate of the link up to $5 \mathrm{Mbps}$, the system delay is 220 ns. 2013, which is located at the frequency $3.9 \mathrm{THz}$ build a wireless communication system. $1 \mathrm{M}$ bps communication rate to achieve $2.4 \mathrm{~m}$ of wireless video transmission [11] [12].

While the above research institutions to build a demonstration millimeter-wave communication systems, and complete the ground a short distance atmospheric attenuation and communication latency test. But no further analysis of the feasibility and demand for satellite space communications.

\section{Summary}

In this work, we give a brief introduction of Satellite Space Information Network, the status of the USA and China are analyzed and compared. There are still much work to do to deal with the space technology compared to the USA. 


\section{Acknowledgements}

This work is funded by China's 973 project under grant of 2012CB316002 and China's 863 project under grant of 2013AA013603, 2012AA011402, National Natural Science Foundation of China(61201192), The Open Research Fund of National Mobile Communications Research Laboratory, Southeast University (2012D02); International Science and Technology Cooperation Program(2012DFG12010); National S \& T Major Project (2013ZX03001024-004), Operation Agreement Between Tsinghua University and Ericsson, Qualcomm Innovation Fellowship, whose funding support is gratefully acknowledgment. The author would also like to thank all the reviewers, their suggestions help improve the work a lot.

\section{References}

[1] $\mathrm{Yi} \mathrm{Xu}$, Shiwen Mao, and Xin Su, "Interference alignment improves the capacity of OFDMA systems," IEEE Transactions on Vehicular Technology, vol.65, no.2, pp.756-767, Feb. 2016. DOI: 10.1109/TVT.2015.2402191.

[2] Gao Y, Zhou W, Ao H, Chu J, Zhou Q, Zhou B, Wang K, Li Y, Xue P. A Novel Optimal Joint Resource Allocation Method in Cooperative Multicarrier Networks: Theory and Practice. Sensors. 2016; 16(4):522

[3] S. Sesia, I. Toufik, and M. Baker, Eds., LTE, The UMTS Long Term Evolution: From Theory to Practice. Wiley, February 2009.

[4] "Satellite Earth Stations and Systems (SES); Satellite Component of UMTS/IMT-2000; General Aspects and Principles,” ETSI, Tech. Rep. 101 865, July 2001.

[5] G. Giambene, S. Giannetti, C. P. Niebla, M. Ries, and A. Sali, "Traffic Management in HSDPA via GEO Satellite,” Space Communications, vol. 21, no. 1-2, pp. 51-68, January 2007.

[6] F. Bastia et al., "LTE Adaptation for Mobile Broadband Satellite Networks," EURASIP Journal on Wireless Communications and Networking, 2009.

[7] V. Cerf et al., "Delay-Tolerant Networking Architecture," Request for Comment 4838, April 2007.

[8] C. Caini and R. Firrincieli, “Tcp Hybla: a TCP Enhancement for Heterogeneous Networks," Int. J. Satell. Commun. Network, vol. 22, no. 5, pp. 547-566, September/October 2004.

[9] C. Caini et al., "Transport Layer Protocols and Architectures for Satellite Networks," International Journal of Satellite Communications and Networking, vol. 25, no. 1, pp. 1-26, 2007.

[10] C. Casetti, M. Gerla, S.Mascolo, M. Y. Sanadidi, and R. Wang, “TCP Westwood: End-to-End Congestion Control for Wired/Wireless Networks," Wireless Networks, vol. 8, no. 4, pp. 467-479, 2002.

[11] I. F. Akyildiz, G. Morabito, and S. Palazzo, "TCP-Peach: a New Congestion Control Scheme for Satellite IP Networks,” IEEE/ACM Transactions on Networking, vol. 9, no. 3, pp. 307-321, 2001.

[12]K. Fall, "A Delay-Tolerant Network Architecture for Challenged Internets," in ACM SIGCOMM'03, Karlsruhe, Germany, August 2003. 